\title{
Modern Attachment Theory: The Central Role of Affect Regulation in Development and Treatment
}

\author{
Judith R. Schore · Allan N. Schore
}

(C) Springer Science+Business Media, LLC 2007

\begin{abstract}
Over the past decade attachment theory has undergone an intense expansion of both its original scientific foundations as well as its applications to clinical work. Bowlby's original description occurred during a period of behaviorism and an emphasis on the strange situation and secure base behaviors, which then gave way to a dominance of cognition and an emphasis on attachment narratives and reflective capacities. We will argue that in line with Bowlby's fundamental goal of the integration of psychological and biological models of human development, the current interest in affective bodily-based processes, interactive regulation, early experience-dependent brain maturation, stress, and nonconscious relational transactions has shifted attachment theory to a regulation theory. This emphasis on the right brain systems that underlie attachment and developmental change has in turn forged deeper connections with clinical models of psychotherapeutic change, all of which are consonant with psychoanalytic understandings. Modern attachment theory can thus be incorporated into the core of social work theory, research, and practice.
\end{abstract}

Keywords Interactive regulation - Affect regulation . Neurobiology $\cdot$ Attachment theory $\cdot$ Relational dynamics

J. R. Schore $(\bowtie)$

The Sanville Institute, Berkeley, USA

e-mail: JRSchore@sbcglobal.net

J. R. Schore - A. N. Schore

9817 Sylvia Ave, Northridge, CA 91324, USA

A. N. Schore

UCLA David Geffen School of Medicine, Los Angeles, USA

e-mail: ANSchore@aol.com

\section{Introduction}

This special edition of the Clinical Social Work Journal affords us a valuable opportunity to put forth our ideas on a modern update of attachment theory, what we call regulation theory, an interdisciplinary developmental model that has specific implications for therapeutic work. Attachment theory is deceptively simple on the surface: it posits that the real relationships of the earliest stages of life indelibly shape our survival functions in basic ways, and that for the rest of the life span attachment processes lie at the center of the human experience. We now can explain in depth why this is so: as a result of interdisciplinary developmental and neurobiological research over the last 15 years Bowlby's core ideas have been expanded into a more complex and clinically relevant model. We will argue that at this point in time, any theory of development and its corresponding theory of therapy must include these psychobiological findings regarding precisely how early emotional transactions with the primary object impact the development of psychic structure, that is, how affective attachment communications facilitate the maturation of brain systems involved in affect and self regulation. The rich intricacy of an integrative interdisciplinary theory now encompasses all the essential elements that allow us to comprehend and treat disorders of self and affect regulation more effectively.

Bowlby's (1969) original descriptions occurred during a period of behaviorism and included an emphasis on the strange situation and secure base behaviors, which then gave way to the dominance of cognition and an emphasis on attachment narratives and reflective capacities. Despite these trends, we remind the reader of Ainsworth's (1969) characterization of Bowlby's seminal Attachment volume: "In effect what Bowlby has attempted is to update 
psychoanalytic theory in the light of recent advances in biology" (p. 998). We suggest that in line with Bowlby's fundamental goal of integrating psychological and biological conceptions of human development, the current clinical and experimental focus on how affective bodilybased attachment processes are nonconsciously interactively regulated within the mother-infant dyad, and how psychobiological attunement and relational stress impact the experience-dependent maturation of early developing brain regulatory systems, has shifted attachment theory to a regulation theory.

The advances in neurobiology initiated in the last decade, the "decade of the brain", have stimulated the transformation of classic attachment theory over the last 10 years. In 1994 Schore offered a large amount of existing interdisciplinary data to propose that attachment communications are critical to the development of structural right brain neurobiological systems involved in processing of emotion, modulation of stress, self-regulation, and thereby the functional origins of the bodily-based implicit self. In 2000, within an introduction to a reissue of Attachment, Schore proposed, "In essence, a central goal of Bowlby's first book is to demonstrate that a mutually enriching dialogue can be organized between the biological and psychological realms" (p. 24), and argued that attachment theory stresses the primacy of affect and is fundamentally a regulation theory. This linkage of the theory with affective dynamics was mirrored in Fonagy et al.'s (2002) Affect Regulation, Mentalization, and the Development of the Self, and Mikulincer et al.'s (2003) work on "attachment theory and affect regulation." Indeed, Fonagy and Target (2002) concluded that "the whole of child development to be the enhancement of self-regulation."

This shift of the theory into affect and affect regulation has had an important effect on translating the developmental theory into a pragmatic framework for models of both psychopathogenesis and the change process in psychotherapy. It is only in the last decade that the clinical applications of attachment theory have been extensively articulated. In parallel to the linkage of early attachment to the neurobiology of optimal and pathological emotional development and the genesis of personality disorders (Schore 2001a, 2002), the problem of linking the theory to psychotherapy models could be elucidated by focusing the treatment upon the affective dynamics of right brain insecure internal working models that are activated within the therapeutic alliance. In the Seventh Annual John Bowlby Lecture, Schore (2001b) proposed that the empathic therapist's capacity to regulate the patient's arousal state within the affectively charged nonconscious transferencecountertransference relationship is critical to clinical effectiveness.
The current energization of modern attachment theory is now being expressed in the updating and deepening of its underlying theoretical concepts, its increased clinical relevance, and its expanded connections with other disciplines (e.g., psychoanalysis, neuroscience, psychiatry, traumatology, pediatrics), including clinical social work. From its very beginnings, attachment theory has shared with clinical social work a common biopsychosocial perspective. Indeed, clinical social work itself is now undergoing a re-examination and re-definition as the quality of master's level education is evaluated (see the March 2007 issue of this journal). We agree with the assertion of Simpson et al. (2007) that the clinical specialization fundamentally includes two core issues, person-in-situation and relationship. The "person-in-situation" orientation encompasses not only nonconscious psychological relational dynamics beginning in infancy, but also individual biological and somatic factors, and social/cultural influences that are both internalized and situational. This biopsychosocial perspective of clinical social work is absolutely consonant with modern attachment theory's elaboration of the mechanisms that operate at the unconscious psychobiological core of the intersubjective context, the brain-mindbody-environment relational matrix out of which each individual emerges. And so we argue that individual development arises out of the relationship between the brain/mind/body of both infant and caregiver held within a culture and environment that supports or threatens it.

To say this in another way, attachment experiences shape the early organization of right brain, the neurobiological core of the human unconscious (Schore 2003b). Clinical social work has long embraced the psychoanalytic concept of the importance of unconscious functions in everyday life. Indeed, therapeutic interventions are rooted in these same dynamic relational processes. The cocreation of an attachment relationship between the empathic social worker and client has also been seen as the sine-qua-non of clinical practice, and respect for the individual is, and always has been, paramount. The current expansion of neurobiologically supported attachment principles of interactive affect communication and regulation both explains and justifies this approach. The mechanisms of developmental change thus include changes in both psychic function and structure, not only in the earliest but also in all subsequent stages of development. Modern attachment theory is thus a regulation theory consonant with the current relational intersubjective trends in the psychodynamic literature, and can thus be readily incorporated into the core of social work theory, research, and practice.

Towards that end, in the following we will outline the general precepts of modern attachment theory, with reference to the practice of clinical social work. We will 
initially present an overview of the central role of unconscious interactive regulation in establishing attachment relationships and the lifelong impact this has on the development of the implicit self. We then discuss the interpersonal neurobiology of implicit nonverbal communications within the therapeutic alliance in the form of transference-countertransference transactions. And finally, we offer some thoughts on the implications of regulation theory for models of clinical expertise. In doing so we will assume a familiarity with basic concepts of classical attachment theory, object-relations, self and relational psychology and focus on integrating these models with their neurobiological underpinnings so that we end up with an interpenetrating and overarching theory.

\section{The Psychobiological Core of Developmental Attachment Communications: Interactive Regulation}

The essential task of the first year of human life is the creation of a secure attachment bond of emotional communication between the infant and the primary caregiver. In order to enter into this communication, the mother must be psychobiologically attuned to the dynamic shifts in the infant's bodily-based internal states of central and autonomic arousal. During the affective communications embedded in mutual gaze episodes the psychobiologically attuned sensitive caregiver appraises nonverbal expressions of the infant's arousal and then regulates these affective states, both positive and negative. The attachment relationship mediates the dyadic regulation of emotion, wherein the mother (primary caregiver) co-regulates the infant's postnatally developing central (CNS) and autonomic (ANS) nervous systems.

In this dialogical process the more the mother contingently tunes her activity level to the infant during periods of social engagement, the more she allows him to recover quietly in periods of disengagement, and the more she attends to his reinitiating cues for reengagement, the more synchronized their interaction. In play episodes of affect synchrony, the pair are in affective resonance, and in such, an amplification of vitality affects and a positive state occurs. In moments of interactive repair the "goodenough" caregiver who has misattuned, can regulate the infant's negative state by accurately re-attuning in a timely manner. The regulatory processes of affect synchrony that create states of positive arousal and interactive repair that modulate states of negative arousal are the fundamental building blocks of attachment and its associated emotions, and resilience in the face of stress and novelty is an ultimate indicator of attachment security. Through sequences of attunement, misattunement, and re-attunement, an infant becomes a person, achieving a "psychological birth"
(Mahler et al. 1975). This preverbal matrix forms the core of the incipient self.

Thus, emotion is initially regulated by others, but over the course of infancy it becomes increasingly self-regulated as a result of neurophysiological development. These adaptive capacities are central to self-regulation, i.e. the ability to flexibly regulate psychobiological states of emotions through interactions with other humans, interactive regulation in interconnected contexts, and without other humans, autoregulation in autonomous contexts. Attachment, the outcome of the child's genetically encoded biological (temperamental) predisposition and the particular caregiver environment, thus represents the regulation of biological synchronicity between and within organisms.

The fundamental role of nonconscious attachment dynamics is therefore interactive psychobiological regulation. According to Pipp and Harmon (1987), "It may be that...we are biologically connected to those with whom we have close relationships...Homeostatic regulation between members of a dyad is a stable aspect of all intimate relationships throughout the lifespan." At the most fundamental level, attachment represents the evolutionary mechanism by which we are sociophysiologically connected to others (Adler 2002), and nonconscious implicit interactive regulation is the central strategy that underlies all essential survival functions of the human self system (Schore 2003a, b).

This principle is echoed in current developmental brain research, where Ovtscharoff and Braun (2001, p. 33) report that "The dyadic interaction between the newborn and the mother...serves as a regulator of the developing individual's internal homeostasis." Notice the similarity to Kohut's (1971) proposal that the infant's dyadic regulatory transactions with the maternal selfobject allow for maintenance of his homeostatic equilibrium. Furthermore, attachment regulatory transactions impact the development of psychic structure, that is, they generate brain development (Schore 1994). In very recent writings Fonagy and Target (2005, p. 334) conclude,

If the attachment relationship is indeed a major organizer of brain development, as many have accepted and suggested (e.g., Schore, 1997, 2003), then the determinants of attachment relationships are important far beyond the provision of a fundamental sense of safety or security (Bowlby 1988).

Even more specifically, the regulatory function of the mother-infant interaction acts as an essential promoter of the development and maintenance of synaptic connections during the establishment of functional circuits of the right brain (Henry 1993; Schore 1994; Sullivan and Gratton 2002). A growing number of studies now support the observation that right lateralized limbic areas responsible 
for the regulation of autonomic functions and higher cognitive processes are involved in the "formation of social bonds" and are "part of the circuitry supporting human social networks," and that the "the strong and consistent predominance for the right hemisphere emerges postnatally" (Allman et al. 2005, p. 367).

Because implicit attachment regulatory functions mature so very early in development, before later forming verbal explicit systems, Schore (1994, 2003a, b) has focused upon the unique operations of the earlier maturing (Chiron et al. 1997) right hemisphere. From infancy throughout all later stages of the lifespan this early evolving right lateralized system is centrally involved in implicit processes and in the control of vital functions supporting survival and enabling the organism to cope with stresses and challenges. He has therefore suggested that the implicit self-system of the right brain that evolves in preverbal stages of development represents the biological substrate of the dynamic unconscious (Schore 2002). Studies in neuroscience now report that this early maturing right hemisphere is centrally involved in "maintaining a coherent, continuous and unified sense of self" (Devinsky 2000), and that a right frontal lobe process, one that connects "the individual to emotionally salient experiences and memories underlying selfschemas, is the glue holding together a sense of self" (Miller et al. 2001). Using functional magnetic resonance imaging Buchheim et al (2006) report that the Adult Attachment Projective activates the right inferior frontal cortex, an area involved in "the control processes involved in emotion regulation".

\section{Right Brain Nonverbal Attachment Communication: The Intersubjective Origins of the Implicit Self}

Schore has described how the hard wiring of the emotion processing limbic circuits of the infant's developing right brain, which are dominant for the emotional sense of self, are influenced by implicit intersubjective affective transactions embedded in the attachment relationship with the mother (Schore 1994, 2005). Implicit processing underlies the quick and automatic handling of non-verbal affective cues in infancy, and "is repetitive, automatic, provides quick categorization and decision-making, and operates outside the realm of focal attention and verbalized experience" (Lyons-Ruth 1999, p. 576). Trevarthen (1990) described how prosodic vocalizations, coordinated visual eye-to-eye messages, and tactile and body gestures, serve as channels of communicative signals in the proto dialogues between infant and mother which induce instant emotional effects. Bowlby (1969) also described "facial expression, posture, and tone of voice" as the essential vehicles of attachment communications between the emerging self and the primary object (Schore 2001a). The dyadic implicit processing of these nonverbal attachment communications are the product of the operations of the infant's right hemisphere interacting with the mother's right hemisphere. Attachment experiences are thus imprinted in an internal working model that encodes strategies of affect regulation that act at implicit nonconscious levels.

Neuroscientists have documented that visual input to the right (and not left) hemisphere during infancy is essential for the development of the capacity to efficiently process information from faces (Le Grand et al. 2003). These findings support earlier speculations in the psychoanalytic literature that "The most significant relevant basic interactions between mother and child usually lie in the visual area: the child's bodily display is responded to by the gleam in the mother's eye" (Kohut 1971, p. 117), that early mental representations are specifically visually oriented (Giovacchini 1981), and that historical visual imagery is derivative of events of early phases of development (Anthi 1983).

With respect to the infant's ability to process the emotional tone of the voice, prosody, it is now thought that:

The right hemisphere of the neonate is actively involved in the perception of speech melody and the intonations of the voices of mother and surrounding people. The pre-speech stage of child development is characterized by interactions of the descriptive and emotional components due mainly to mechanisms operating within the hemispheres on the principle of non-verbal communication (Bogolepova and Malofeeva 2001, p. 353).

And on the other side of the right brain-to-right brain communication system within the attachment dyad, researchers describe the mother's processing capacities: "A number of functions located within the right hemisphere work together to aid monitoring of a baby. As well as emotion and face processing the right hemisphere is also specialized in auditory perception, the perception of intonation, attention, and tactile information" (Bourne and Todd 2004, pp. 22-23).

It is important to note that these early experiences may be regulated or dysregulated, imprinting either secure or insecure attachments. Watt (2003, p. 109) observes, "If children grow up with dominant experiences of separation, distress, fear and rage, then they will go down a bad pathogenic developmental pathway, and it's not just a bad psychological pathway but a bad neurological pathway." This is due to the fact that during early critical periods organized and disorganized insecure attachment histories are "affectively burnt in" the infant's rapidly developing right brain (Schore 2001a, 2003a). These stressful 
relational experiences are encoded in unconscious internal working models in the right, and not left, brain. In a study of hemispheric lateralization of avoidant attachment, Cohen and Shaver (2004) conclude "Emotional negativity and withdrawal motivation have been connected in psychophysiological studies with the right frontal lobe of the brain" (p. 801), and that avoidant individuals show "a right hemisphere advantage for processing negative emotion and attachment-related words" (p. 807).

Summarizing a large body of neuropsychological data Feinberg and Keenan (2005) conclude:

The right hemisphere, particularly the right frontal region, under normal circumstances plays a crucial role in establishing the appropriate relationship between the self and the world...dysfunction results in a two-way disturbance of personal relatedness between the self and the environment that can lead to disorders of both under and over relatedness between the self and the world (p.15).

In relationally-oriented therapeutic contexts that optimize intersubjective communication and interactive regulation, deficits in internal working models of the self and the world are gradually repaired. Recall, Bowlby (1988) asserted the restoring into consciousness and reassessment of internal working models is the essential task of psychotherapy.

Decety and Chaminade's (2003) characterization of higher right brain functions is directly applicable to psychotherapy of disorders of the self:

Mental states that are in essence private to the self may be shared between individuals...self-awareness, empathy, identification with others, and more generally intersubjective processes, (and) are largely dependent upon...right hemisphere resources, which are the first to develop (p. 591).

These particular implicit right brain operations are essential for adaptive interpersonal functioning, and are specifically activated in the therapeutic alliance. Right brain increases in "implicit relational knowledge" stored in the nonverbal domain (Stern et al. 1998) thus lie at the core of the psychotherapeutic change process.

As the right hemisphere is also dominant for the broader aspects of communication and for subjective emotional experiences, the implicit communication of affective states between the right brains of the members of the infantmother and patient-therapist dyads is thus best described as "intersubjectivity." The neurobiological correlate of this intersubjectivity principle is expressed in the dictum, "the self-organization of the developing brain occurs in the context of a relationship with another self, another brain" (Schore 1996). This is true in both the developmental and therapeutic growth-facilitating contexts. The interpersonal neurobiology of modern attachment theory has thus been a rich source of information about the essential role of nonconscious nonverbal right communications in the psychotherapy relationship.

\section{Right Brain Nonverbal Attachment Communication: Implicit Communications Within the Therapeutic Alliance}

It is now accepted that the "non-verbal, prerational stream of expression that binds the infant to its parent continues throughout life to be a primary medium of intuitively felt affective-relational communication between persons" (Orlinksy and Howard 1986). Right brain transactions also mediate the relational unconscious as it is expressed in the psychoanalytic encounter, and Lyons-Ruth's (2000) description of affective exchanges of implicit relational knowledge within the therapeutic alliance:

Most relational transactions rely heavily on a substrate of affective cues that give an evaluative valence or direction to each relational communication. These occur at an implicit level of rapid cueing and response that occurs too rapidly for simultaneous verbal transaction and conscious reflection. (pp. 91-92).

Scaer (2005) describes essential implicit communications embedded within the therapist-client relationship:

Many features of social interaction are nonverbal, consisting of subtle variations of facial expression that set the tone for the content of the interaction. Body postures and movement patterns of the therapist...also may reflect emotions such as disapproval, support, humor, and fear. Tone and volume of voice, patterns and speed of verbal communication, and eye contact also contain elements of subliminal communication and contribute to the unconscious establishment of a safe, healing environment (pp. 167-168).

These right brain communications convey expressions of "the personality of the therapist" more so than conscious verbalizations.

These developmental studies have direct relevance to the psychotherapeutic process based on the commonality of implicit intersubjective right brain-to-right brain emotion transacting and regulating mechanisms in the caregiverinfant relationship and the therapist-patient relationship. Schore describes the nature of implicit and explicit processes in the psychotherapeutic context: 
During the treatment, the empathic therapist is consciously, explicitly attending to the patient's verbalizations in order to objectively diagnose and rationalize the patient's dysregulating symptomatology. But she is also listening and interacting at another level, an experience-near subjective level, one that implicitly processes moment-to-moment socioemotional information at levels beneath awareness (Schore 2003b, p. 52).

A fundamental question of treatment is how we work with what is being communicated but not symbolized with words. In discussing subsymbolic processing, Bucci (2002) observes "We recognize changes in emotional states of others based on perception of subtle shifts in their facial expression or posture, and recognize changes in our own states based on somatic or kinesthetic experience (p. 194)." These implicit communications between the client and therapist's right brain systems are expressed within the therapeutic alliance between the client and therapist's right brain systems.

Human beings rely extensively on nonverbal channels of communication in their day-to-day emotional as well as interpersonal exchanges. The verbal channel, language, is a relatively poor medium for expressing the quality, intensity and nuancing of emotion and affect in different social situations...the face is thought to have primacy in signaling affective information. (Mandal and Ambady 2004, p. 23)

As in the developmental attachment context, right brainto-right brain prosodic communications also act as an essential vehicle of implicit communications within the therapeutic relationship. The right hemisphere is important in the processing of the "music" behind our words. When listening to speech, we rely upon a range of cues upon which to base our inference as to the communicative intent of others. To interpret the meaning of speech, how something is said is as important as what is actually said. Prosody conveys different shades of meaning by means of variations in stress and pitch-irrespective of the words and grammatical construction (Mitchell et al. 2003). These data support suggestions that the preverbal elements of language-intonation, tone, force, and rhythm-stir up reactions derived from the early mother-child relationships (Greenson 1978). In the recent literature on the psychotherapeutic context, Andrade concludes, "It is the affective content of the analyst's voice-and not the semantic content-that has an impact on the patient's store of implicit memories" (2005, p. 683).

During heightened affective moments these right brain dialogues between the relational unconscious of both the patient and therapist (like the attachment communications of the infant and mother) are examples of "primary process communication" (Dorpat 2001). According to this author, "The primary process system analyzes, regulates, and communicates an individual's relations with the environment":

[A]ffective and object-relational information is transmitted predominantly by primary process communication. Nonverbal communication includes body movements (kinesics), posture, gesture, facial expression, voice inflection, and the sequence, rhythm, and pitch of the spoken words (Dorpat 2001, p. 451).

Interestingly, in addition to psychoanalytic authors who have implicated the right brain in primary process functions (see Schore 1994), neuroscience researchers now contend that "the right hemisphere operates in a more free-associative, primary process manner, typically observed in states such as dreaming or reverie." (Grabner et al., p. 228).

It is important to stress that all of these implicit nonconscious right brain/mind/body nonverbal communications are bidirectional and thereby intersubjective (see Schore 2003b for a right hemisphere-to-right hemisphere model of projective identification, a fundamental process of implicit communication within the therapeutic alliance). Meares (2005) describes,

Not only is the therapist being unconsciously influenced by a series of slight and, in some cases, subliminal signals, so also is the patient. Details of the therapist's posture, gaze, tone of voice, even respiration, are recorded and processed. A sophisticated therapist may use this processing in a beneficial way, potentiating a change in the patient's state without, or in addition to, the use of words (p. 124).

Implicit right brain-to-right brain intersubjective transactions lie at the core of the therapeutic relationship. They mediate what Sander (1992) calls "moments of meeting" between patient and therapist. In light of current neurobiological data that suggests "While the left hemisphere mediates most linguistic behaviors, the right hemisphere is important for broader aspects of communication" (van Lancker and Cummings 1999), Schore (2003b) has proposed that just as the left brain communicates its states to other left brains via conscious linguistic behaviors so the right nonverbally communicates its unconscious states to other right brains that are tuned to receive these communications. Regulation theory thus describes how implicit systems of the therapist interact with implicit systems of the patient; psychotherapy is not the "talking" but the "communicating" cure. 


\section{Transference-Countertransference as Implicit Right Brain/Mind/Body Transactions}

Advances in neuroscience now clearly suggest that the capacity to receive and express communications within the implicit realm is optimized when the clinician is in a state of right brain receptivity. Marcus (1997) observes, "The analyst, by means of reverie and intuition, listens with the right brain directly to the analysand's right brain (p. 238)." The neuroscience literature holds that "The left hemisphere is more involved in the foreground-analytic (conscious) processing of information, whereas the right hemisphere is more involved in the background-holistic (subconscious) processing of information" (Prodan et al. 2001, p. 211).

Indeed, the right hemisphere uses an expansive attention mechanism that focuses on global features while the left uses a restricted mode that focuses on local detail (Derryberry and Tucker 1994). In contrast to the left hemisphere's activation of "narrow semantic fields", the right hemisphere's "coarse semantic coding is useful for noting and integrating distantly related semantic information" (Beeman 1998), a function which allows for the process of free association. Bucci (1993) has described free association as following the tracks of nonverbal schemata by loosening the hold of the verbal system on the associative process and giving the nonverbal mode the chance to drive the representational and expressive systems, that is by shifting dominance from a left to right hemispheric state.

These nonverbal affective and thereby mind/body communications are expressions of the right brain, which is centrally involved in the analysis of direct kinesthetic information received by the subject from his own body, an essential implicit process. This hemisphere, and not the linguistic, analytic left, contains the most comprehensive and integrated map of the body state available to the brain (Damasio 1994). The therapist's right hemisphere allows her to know the patient "from the inside out" (Bromberg 1991, p. 399). To do this the clinician must access her own bodily-based intuitive responses to the patient's communications. In an elegant description Mathew's (1998) evocatively portrays this omnipresent implicit process of bodily communications:

The body is clearly an instrument of physical processes, an instrument that can hear, see, touch and smell the world around us. This sensitive instrument also has the ability to tune in to the psyche: to listen to its subtle voice, hear its silent music and search into its darkness for meaning (p. 17).

Intersubjectivity is thus more than a match or communication of explicit cognitions. The intersubjective field co-constructed by two individuals includes not just two minds but two bodies (Schore 1994, 2003a, b). At the psychobiological core of the intersubjective field is the attachment bond of emotional communication and interactive regulation. Recall Pipp and Harmon's (1987) assertion that the fundamental role of nonconscious attachment dynamics is interactive regulation. Implicit unconscious intersubjective communications are interactively communicated and regulated and dysregulated psychobiological somatic processes that mediate shared conscious and unconscious emotional states, not just mental contents. The essential biological purpose of intersubjective communications in all human interactions, including those embedded in the psychobiological core of the therapeutic alliance, is the regulation of right brain/ mind/body states. These ideas resonate with Shaw's (2004) conclusion,

Psychotherapy is an inherently embodied process. If psychotherapy is an investigation into the intersubjective space between client and therapist, then as a profession we need to take our bodily reactions much more seriously than we have so far because...the body is "the very basis of human subjectivity." (p. 271)

There is now a growing consensus that despite the existence of a number of distinct theoretical perspectives in clinical work, the concepts of transference and countertransference represent a common ground. In a neuropsychological description that echoes psychoanalytic conceptions of transference Shuren and Grafman (2002) propose,

The right hemisphere holds representations of the emotional states associated with events experienced by the individual. When that individual encounters a familiar scenario, representations of past emotional experiences are retrieved by the right hemisphere and are incorporated into the reasoning process (p. 918).

Transference-countertransference transactions thus represent nonconscious nonverbal right brain-mind-body communications. Transference has been described as "an expression of the patient's implicit perceptions and implicit memories" (Bornstein 1999). Facial indicators of transference are expressed in visual and auditory affective cues quickly appraised from therapist's face. Countertransference is similarly currently defined in nonverbal implicit terms as the therapist's "autonomic responses that are reactions on an unconscious level to nonverbal messages" (Jacobs 1994). In monitoring countertransferential responses the clinician's right brain tracks at a preconscious level not only the arousal rhythms and flows of the patient's affective states, but also her own interoceptive bodilybased affective responses to the patient's implicit facial, gestural, and prosodic communications. 
It is certainly true that the clinician's left-brain conscious mind is an important contributor to the treatment process. But perhaps more than other treatment modalities, psychodynamic psychotherapeutic models have focused upon the critical functions of the therapist's "unconscious right mind." The right hemisphere plays a dominant role in the processing of self-relevant information (Molnar-Szakacs et al. 2005), affective theory of mind (Schore 2003b), empathy (Schore 1994; Shamay-Tsoory et al. 2003), as well as in mentalizing (Ohnishi et al. 2004). A neuropsychoanalytic right brain perspective of the treatment process allows for a deeper understanding of the critical factors that operate at implicit levels of the therapeutic alliance, beneath the exchanges of language and explicit cognitions.

In this intersubjective dialogue, the psychobiologically attuned, intuitive clinician, from the first point of contact, is learning the nonverbal moment-to-moment rhythmic structures of the client's internal states, and is relatively flexibly and fluidly modifying her own behavior to synchronize with that structure, thereby co-creating with the client a growth-facilitating context for the organization of the therapeutic alliance. The attachment between therapist and client is established over time, allowing for the expression of experiences that resonate with the original infant-mother intersubjective history of the first 2 years. If that was an insecure attachment to begin with, co-creating a new, secure interaction will take even longer.

Over the ensuing stages of the treatment, the sensitive empathic clinician's monitoring of unconscious process rather than content calls for right brain attention to her matching the patient's implicit affective-arousal states. The empathic therapist also resonates with the client's simultaneous implicit expressions of engagement and disengagement within the co-constructed intersubjective field. This in turn allows the clinician to act as an interactive regulator of the patient's psychobiological states. Such work implies a profound commitment by both participants in the therapeutic dyad and a deep emotional involvement on the part of the therapist (Tutte 2004). Ultimately, effective psychotherapeutic treatment of early evolving self-pathologies (severe personality disorders) facilitates changes in complexity of the right hemispheric unconscious system.

\section{Further Implications of Regulation Theory for Clinical Social Work: Models of Clinical Expertise}

The unique biopsychosocial perspective of clinical social work highlights the critical importance of unconscious forces that drive all human emotion, cognition, and behavior within a sociocultural matrix. From its beginnings, attachment theory, grounded in psychoanalysis and ethology, has focused on how real experiences, especially in childhood, directly impact the unconscious system. This is, of course, a core principle of psychodynamically oriented clinical social work. This updated form of attachment theory, that is informed by neuroscience, elucidates the early experience-dependent development of the human unconscious system, which remains active over the course of the life span. In an excellent volume, Neurobiology for Clinical Social Work, Applegate and Shapiro (2005) apply attachment neurobiology specifically to social work practice.

We suggest that clinical expertise, especially with severely disturbed patients, relies more on nonconscious nonverbal right brain than conscious verbal left brain functions. Clinical efficacy is more than explicit left hemispheric technical skill in interpretation. Rather, increasing levels of clinical effectiveness with a broader spectrum of patients fundamentally involves more complex learning of a number of nonconscious functions of the therapist's right brain that are expressed in the therapeutic alliance. All technique sits atop these right brain implicit skills, which deepen and expand with clinical experience: the ability to receive and express nonverbal affective communications; clinical sensitivity; use of subjectivity/ intersubjectivity; empathy; and affect regulation. Neuroscience now indicates that the implicit processes of intuition (Allman et al. 2005), creativity (Grabner et al. 2007), and indeed insight (Jung-Beeman et al. 2004), are all right, and not left, brain functions.

A Presidential Task Force on Evidence-Based Practice prepared by the American Psychological Association (2006) now suggests,

Central to clinical expertise is interpersonal skill, which is manifested in forming a therapeutic relationship, encoding and decoding verbal and nonverbal responses, creating realistic but positive expectations, and responding empathically to the patient's explicit and implicit experiences and concerns (p. 277).

They further note that "Research suggests that sensitivity and flexibility in the administration of therapeutic interventions produces better outcomes than rigid application of...principles" (p. 278).

Sensitivity has, of course, been well-studied in the developmental attachment literature, where researchers observe that maternal sensitivity cultivates synchronous, reciprocal, and jointly satisfying mother-infant interactions, which, in turn, foster the development of a secure attachment relationship. In adult attachment studies Schachner et al. (2005) argue "nonverbal behavior and sensitivity to a relationship partner's nonverbal behavior importantly influence the quality of interpersonal 
interactions and relationships, including attachment relationships." We suggest that this attachment principle applies to the therapeutic relationship as well.

The dictionary definition of sensitivity is: "susceptible to the attitudes, feelings, or circumstances of others; registering very slight differences or changes of emotion (American Heritage Dictionary)". In previous writings Schore (2003b) describes the operations of the therapist's right brain by which "the sensitive clinician's oscillating attentiveness is focused on barely perceptible cues that signal a change in state and on nonverbal behaviors and shifts in affects." In discussing "the art of psychotherapy" Bugental (1987) stresses the importance of the sensitive clinician's ability to "learn to experience finer and finer distinctions or nuances." He states, "The primary instrument brought to the support of the client's therapeutic efforts is the therapist's trained, practiced, and disciplined sensitivity. In many ways, this sensitivity is akin to a musical instrument which must be carefully prepared, maintained, tuned, and protected" (p. 222). The clinician's capacity for intersubjective communication depends upon her "being open to intuitive sensing of what is happening in the back of the patient's words and, often, back of his conscious awareness" (p. 11).

This clinical sensitivity to even low levels of nonverbal attachment communications allows for the clinician's involvement in a wider array of co-created affectively charged intersubjective fields. These collaborations of the client and therapist's subjectivities allows for right brain communications and regulations of dysregulated affective states. The importance of this connection is stressed by Whitehead (2006):

[E]very time we make therapeutic contact with our patients we are engaging profound processes that tap into essential life forces in our selves and in those we work with...Emotions are deepened in intensity and sustained in time when they are intersubjectively shared. This occurs at moments of deep contact (p. 624).

An attachment-based clinical approach highlights the unconscious nonverbal affective more than the conscious verbal cognitive factors as the essential change process of psychotherapy. Thus, at the most fundamental level, the intersubjective work of psychotherapy is not defined by what the therapist does for the patient, or says to the patient (left brain focus). Rather, the key mechanism is how to be with the patient, especially during affectively stressful moments (right brain focus).

Bowlby stated that attachment behavior was based on the need for safety and a secure base. We have demonstrated that attachment is more than this; it is the essential matrix for creating a right brain self that can regulate its own internal states and external relationships. Attachment intersubjectivity allows psychic structure to be built and shaped into a unique human being. Our task as therapists is to understand and facilitate this developmental process with our clients. As clinical social workers we do this in the wider context of the culture and society. Regulation theory enhances and deepens the field's bio-psycho-social-cultural perspective.

\section{Conclusion: Modern Regulation Theory}

An explosion of developmental and neurobiological research has added substantially to the theoretical understanding of the 110 years since Freud (1895) first published his Project for a Scientific Psychology (Schore 1997). Having been grounded in drive, ego, object-relations, self and relational psychology through the 1980s, the addition of attachment theory has moved psychodynamic clinician's sensibilities into an awareness of real experience and a keen focus on early development as the root of all. Then, beginning in the 1990s, the advances in neuroscience, added to research on temperament, the biological component in our biopsychosocial frame, has provided a remarkable underpinning and expansion of all the pertinent developmental psychoanalytic theoretical concepts that came before. Using this knowledge on a daily basis, finding new understandings in clinical assessments, shaping therapeutic interventions from relevant theory, and providing a unique awareness of the adaptive nonconscious functions of the implicit self are some of the profound results of this theoretical integration.

Thus, we are proposing the concept of regulation theory as an amalgam of Bowlby's attachment theory, updated internal object relations theories, self psychology, and contemporary relational theory all informed by neuroscience and infant research. This is a profoundly developmental approach. We understand any individual's personal trajectory of emotional growth, including the development of his/her unconscious, to be facilitated or inhibited by the context of his/her family and culture. Attachment outcomes are thus the product of the interactions of both nature and nurture, the strengths and weaknesses of the individual's genetically encoded biological predispositions (temperament) and the early dyadic relationships with caregivers embedded within a particular social environment (culture).

The developmental understanding that arises from this theory leads to a corresponding regulation theory of therapy. This therapeutic approach is rooted in an awareness of the centrality of early dyadic regulation, a thorough knowledge of right hemispheric emotional development, and a deep understanding of the dynamics of implicit 
procedural memory. An understanding of the right brain mechanisms that underlie bodily-based non-verbal communication is essential in this approach. A keen apperception of one's own somatic countertransference is a key element in the intersubjectivity between therapist and client. We know the effects of stressors on the self system, from mild and "ordinary" peculiarities that create and shape individuality, to severe trauma and neglect that interfere with and derail normal development and that require long-term therapeutic involvement to get back on track (Schore 2002).

Regulation theory explains how these "external" developmental and therapeutic attachment experiences are transformed into "internal" regulatory capacities. And we know from research that this intensive therapeutic relationship can repair damage and create new structure that is more able to cope with the demands of life. The intersubjective process of developing a resilient self that can enter into a variety of meaningful relationships shows us how the internal world is structured on a psychophysiological base that takes into account the unique genetic endowment of the particular infant in interaction with his relational environment. The psychotherapeutic process is based on this dynamic and can act as a growth facilitating social environment that can promote the development of not only an "earned secure" attachment, but expansion of the right brain human unconscious.

The regulation model of modern attachment theory has implications not only for social work's important role in the psychotherapeutic treatment of individuals, but also for the culture, an area of prime interest to social work. Tucker (1992) observes: "the baby brain must begin participating effectively in the process of social information transmission that offers entry into the culture." He asserts that social interaction that promotes brain differentiation is the mechanism for teaching "the epigenetic patterns of culture," and that successful social development requires a high degree of skill in negotiating emotional communication, "much of which is nonverbal." Tucker concludes that such emotional information engages 'specialized neural networks in humans, within the right hemisphere." These data clearly imply an important role for clinical social work in infant mental health and optimal right brain development, that is, attachment programs of prevention and early intervention. The field could make important contributions towards the creation of more emotionally intelligent future cultures.

\section{References}

Adler, H. M. (2002). The sociophysiology of caring in the doctorpatient relationship. Journal of General Internal Medicine, 17, 883-890.

Ainsworth, M. D. S. (1969). Object relations, dependency and attachment: A theoretical review of the infant-mother relationship. Child Development, 40, 969-1025.
Allman, J. M., Watson, K. K., Tetreault, N. A., \& Hakeem, A. Y. (2005). Intuition and autism: A possible role for Von Economo neurons. Trends in Cognitive Sciences, 9, 367-373.

Andrade, V. M. (2005). Affect and the therapeutic action in psychoanalysis. International Journal of Psychoanalysis, 86, 677-697.

Anthi, P. R. (1983). Reconstruction of preverbal experience. Journal of the American Psychoanalytic Association, 31, 33-58.

APA Presidential Task Force on Evidence-Based Practice (2006). Evidence-based practice in psychology. American Psychologist, 61, 271-285.

Applegate, J. S., \& Shapiro, J. R. (2005). Neurobiology for clinical social work: Theory and practice. New York: WW Norton.

Beeman, M. (1998). Coarse semantic coding and discourse comprehension. In M. Beeman \& C. Chiarello (Eds.), Right hemisphere language comprehension. Mahweh, $\mathrm{NJ}$ : Erlbaum.

Bogolepova, I. N., \& Malofeeva, L. I. (2001). Characteristics of the development of speech motor areas 44 and 45 in the left and right hemispheres of the human brain in early post-natal ontogenesis. Neuroscience and Behavioral Physiology, 31, 13-18.

Bornstein, R. F. (1999), Source amnesia, misattribution, and the power of unconscious perceptions and memories. Psychoanalytic Psychology, 16, 155-178.

Bourne, V. J., \& Todd, B. K. (2004). When left means right: An explanation of the left cradling bias in terms of right hemisphere specializations. Developmental Science, 7, 19-24.

Bowlby, J. (1969). Attachment and loss. Vol. 1: Attachment. New York: Basic Books.

Bowlby, J. (1988). A secure base (2nd ed.). New York: Basic Books.

Bromberg, P. M. (1991). On knowing one's patient inside out: The aesthetics of unconscious communication. Psychoanalytic Dialogues, 1, 399-422.

Bucci, W. (1993). The development of emotional meaning in free association: A multiple code theory. In A. Wilson \& J. E. Gedo (Eds.), Hierarchical concepts in psychoanalysis. New York: Guilford Press.

Bucci, W. (2002). The referential process, consciousness, and the sense of self. Psychoanalytic Inquiry, 5, 766-793.

Buchheim, A., Erk, S., George, C., Kachele, H., Ruchsow, M., Spitzer, M., Kircher, T., \& Walter, H. (2006). Measuring attachment representation in an fMRI environment: A pilot study. Psychopathology, 39, 144-152.

Bugental, J. F. (1987). The art of the psychotherapist. New York: W. W. Norton.

Chiron, C., Jambaque, I., Nabbout, R., Lounes, R., Syrota, A., \& Dulac, O. (1997). The right brain hemisphere is dominant in human infants. Brain, 120, 1057-1065.

Cohen, M. X., \& Shaver, P. R. (2004). Avoidant attachment and hemispheric lateralization of the processing of attachment- and emotion-related words. Cognition and Emotion, 18, 799-814.

Damasio, A. R. (1994). Descartes' error. New York: Grosset/Putnam.

Decety, J., \& Chaminade, T. (2003). When the self represents the other: A new cognitive neuroscience view on psychological identification. Consciousness and Cognition, 12, 577-596.

Derryberry, D., \& Tucker, D. M. (1994). Motivating the focus of attention. In P. M. Niedentahl \& S. Kiyayama (Eds.), The heart's eye: Emotional influences in perception and attention. San Diego: Academic Press.

Devinsky, O. (2000). Right cerebral hemispheric dominance for a sense of corporeal and emotional self. Epilepsy \& Behavior, $1,60-73$.

Dorpat, T. L. (2001). Primary process communication. Psychoanalytic Inquiry, 3, 448-463.

Feinberg, T. E., \& Keenan, J. P. (2005). Where in the brain is the self? Consciousness and Cognition, 14, 661-678. 
Fonagy, P., Gergely, G., Jurist, E. L., \& Target, M. (2002). Affect regulation, mentalization and the development of the self. New York: Other Press.

Fonagy, P, \& Target M. (2002). Early intervention, the development of self-regulation. Psychoanalytic Inquiry, 22, 307-335.

Fonagy, P., \& Target, M. (2005). Bridging the transmission gap: An end to an important mystery of attachment research? Attachment \& Human Development, 7, 333-343.

Freud, S. (1895). Project for a scientific psychology. Standard Edition, 1, 295-397. London: Hogarth Press, 1966.

Giovacchini, P. I. (1981). Object relations, deficiency states, and the acquisition of psychic structure. In S. Tutman, C. Kaye, \& M. Zimmerman (Eds.), Object and self: A developmental approach (pp. 397-427). New York: International Universities Press.

Grabner, R. H., Fink, A., \& Neubauer, A. C. (2007). Brain correlates of self-related originality of ideas: Evidence from event-related power and phase-locking changes in the EEG. Behavioral Neuroscience, 121, 224-230.

Greenson, R. (1978). Explorations in psychoanalysis. New York: International Universities Press.

Henry, J. P. (1993). Psychological and physiological responses to stress: The right hemisphere and the hypothalamo-pituitaryadrenal axis, an inquiry into problems of human bonding. Integrative Physiological and Behavioral Science, 28, 369-387.

Jacobs, T. J. (1994). Nonverbal communications: Some reflections on their role in the psychoanalytic process and psychoanalytic education. Journal of the American Psychoanalytic Association, 42, 741-762.

Jung-Beeman, M. et al. (2004). Neural activity when people solve verbal problems with insight. PLoS Biology, 2, 500-510.

Kohut, H. (1971). The analysis of the self. New York: International University Press.

Le Grand, R., Mondloch, C., Maurer, D., \& Brent, H. P. (2003). Expert face processing requires visual input to the right hemisphere during infancy. Nature Neuroscience, 6, 1108-1112.

Lyons-Ruth, K. (1999). The two-person unconscious: Intersubjective dialogue, enactive relational representation, and the emergence of new forms of relational organization. Psychoanalytic Inquiry, $19,576-617$.

Lyons-Ruth, K. (2000). "I sense that you sense that I sense...": Sander's recognition process and the specificity of relational moves in the psychotherapeutic setting. Infant Mental Health Journal, 21, 85-98.

Mahler, M., Pine, F., \& Bergman, A. (1975). The psychological birth of the human infant. New York: Basic Books.

Mandal, M. K., \& Ambady, N. (2004). Laterality of facial expressions of emotion: Universal and culture-specific influences. Behavioral Neurology, 15, 23-34.

Marcus, D. M. (1997). On knowing what one knows. Psychoanalytic Quarterly, 66, 219-241.

Mathew, M. (1998). The body as instrument. Journal of the British Association of Psychotherapists, 35, 17-36.

Meares, R. (2005), The metaphor of play. Origin and breakdown of personal being, 3rd ed. London: Routledge.

Mikulincer, M., Shaver, P. R., \& Pereg, D. (2003). Attachment theory and affect regulation: The dynamics, development, and cognitive consequences of attachment-related strategies. Motivation and Emotion, 27, 77-102. .

Miller, B. L., Seeley, W. W., Mychack, P., Rosen, H. J., Mena, I., \& Boone, K. (2001). Neuroanatomy of the self. Evidence from patients with frontotemporal dementia. Neurology, 57, 817-821.

Mitchell, R. L. C., Elliott, R., Barry, M., Crittenden, A., \& Woodruff, P. W. R. (2003). The neural response to emotional prosody, as revealed by functional magnetic resonance imaging. Neuropsychologia, 41, 1410-1421.
Molnar-Szakacs, I., Uddin, L. Q., \& Iacoboni, M. (2005). Righthemisphere motor facilitation by self-descriptive personalitytrait words. European Journal of Neuroscience, 21, 2000-2006.

Ohnishi, T., et al. (2004). The neural network for the mirror system and mentalizing in normally developed children: An fMRI study. NeuroReport, 15, 1483-1487.

Orlinsky, D. E., \& Howard, K. I. (1986), Process and outcome in psychotherapy. In S. L. Garfield \& A. E. Bergin (Eds.), Handbook of psychotherapy and behavior change (3rd ed.). New York: Wiley.

Ovtscharoff, W. Jr., \& Braun, K. (2001). Maternal separation and social isolation modulate the postnatal development of synaptic composition in the infralimbic cortex of octodon degus. Neuroscience, 104, 33-40.

Pipp, S., \& Harmon, R. J. (1987). Attachment as regulation: A commentary. Child Development, 58, 648-652.

Prodan, C. I., Orbelo, D. M., Testa, J. A., \& Ross, E. D. (2001). Hemispheric differences in recognizing upper and lower facial displays of emotion. Neuropsychiatry, Neuropsychology and Behavioral Neurology, 14, 206-212.

Sander, L. (1992). Letter to the editor. International Journal of Psychoanalysis, 73, 582-584.

Scaer, R. (2005). The trauma spectrum: Hidden wounds and human resiliency. New York: W. W. Norton.

Schachner, D. A., Shaver, P. R., \& Mikulincer, M. (2005). Patterns of nonverbal behavior and sensitivity in the context of attachment relationships. Journal of Nonverbal Behavior, 29, 141-169.

Schore, A. N. (1994). Affect regulation and the origin of the self. Mahweh, NJ: Erlbaum.

Schore, A. N. (1996). The experience-dependent maturation of a regulatory system in the orbital refrontal cortex and the origin of developmental psychopathology. Development and Psychopathology, 8, 59-87.

Schore, A. N. (1997). A century after Freud's Project: Is a rapprochement between psychoanalysis and neurobiology at hand? Journal of the American Psychoanalytic Association, 45, 841-867.

Schore, A. N. (2000). Attachment and the regulation of the right brain. Attachment \& Human Development, 2, 23-47.

Schore, A. N. (2001a). The effects of a secure attachment relationship on right brain development, affect regulation, and infant mental health. Infant Mental Health Journal, 22, 7-66.

Schore, A. N. (2001b). The Seventh Annual John Bowlby Memorial Lecture, Minds in the making: Attachment, the self-organizing brain, and developmentally-oriented psychoanalytic psychotherapy. British Journal of Psychotherapy, 17, 299-328.

Schore, A. N. (2002). Advances in neuropsychoanalysis, attachment theory, and trauma research: Implications for self psychology. Psychoanalytic Inquiry, 22, 433-484.

Schore, A. N. (2003a). Affect dysregulation and disorders of the self. New York: W. W. Norton.

Schore, A. N. (2003b). Affect regulation and the repair of the self. New York: W. W. Norton.

Schore, A. N. (2005). Attachment, affect regulation, and the developing right brain: Linking developmental neuroscience to pediatrics. Pediatrics In Review, 26, 204-211.

Shamay-Tsoory, S. G., Tomer, R., Berger, B. D., \& Aharon-Peretz, J. (2003). Characterization of empathy deficits following prefrontal brain damage: The role of the right ventromedial prefrontal cortex. Journal of Cognitive Neuroscience, 15, 324-337.

Shaw, R. (2004). The embodied psychotherapist: An exploration of the therapists' somatic phenomena within the therapeutic encounter. Psychotherapy Research, 14, 271-288.

Shuren, J. E., \& Grafman, J. (2002). The neurology of reasoning. Archives of Neurology, 59, 916-919. 
Simpson, G., Williams, J., \& Segall, A. (2007). Social work education and clinical learning. Clinical Social Work Journal, 35, 3-14.

Stern, D. N., Bruschweiler-Stern, N., Harrison, A. M., Lyons-Ruth, K., Morgan, A. C., Nahum, J. P., Sander, L., \& Tronick, E. Z. (1998). The process of therapeutic change involving implicit knowledge: Some implications of developmental observations for adult psychotherapy. Infant Mental Health Journal, 19, 300-308.

Sullivan, R. M., \& Gratton, A. (2002). Prefrontal cortical regulation of hypothalamic-pituitary-adrenal function in the rat and implications for psychopathology: Side matters. Psychoneuroendocrinology, 27, 99-114.

Trevarthen, C. (1990). Growth and education of the hemispheres. In C. Trevarthen (Ed.), Brain circuits and functions of the mind (pp. 334-363). Cambridge, England: Cambridge University Press.

Tucker, D. M. (1992). Developing emotions and cortical networks. In M. R. Gunnar \& C. A. Nelson (Eds.), Minnesota symposium on child psychology. Vol. 24, Developmental behavioral neuroscience (pp. 75-128). Hillsdale, NJ: Erlbaum.

Tutte, J. C. (2004). The concept of psychical trauma: A bridge in interdisciplinary space. International Journal of Psychoanalysis, 85, 897-921.

van Lancker, D., \& Cummings, J. L. (1999). Expletives: Neurolingusitic and neurobehavioral perspectives on swearing. Brain Research Reviews, 31, 83-104.
Watt, D. F. (2003). Psychotherapy in an age of neuroscience: Bridges to affective neuroscience. In J. Corrigall \& H. Wilkinson (Eds.), Revolutionary connections. Psychotherapy and neuroscience (pp. 79-115). Karnac: London.

Whitehead, C. C. (2006). Neo-psychoanalysis: A paradigm for the 21st century. Journal of the Academy of Psychoanalysis and Dynamic Psychiatry, 34, 603-627.

\section{Author Biographies}

Judith R. Schore Ph.D. is Dean of Students and Core Faculty at The Sanville Institute. She teaches Attachment and Neurobiology at ReissDavis Child Center's Child Psychotherapy training Program. She has had private psychotherapy practice in Northridge, California for over 30 years. She is currently co-editing a volume called "A Reader's Guide to Interpersonal Neurobiology" to be published by Norton.

Allan N. Schore Ph.D. is on the clinical faculty at UCLA School of Medicine and Center for Culture, Brain, and Development. He is author of Affect Regulation and the Origin of the Self, Affect Dysregulation and Disorders of the Self and Affect Regulation and the Repair of the Self, as well as numerous articles and chapters. He has had private psychotherapy practice in Northridge, California for over 30 years. He is currently co-editing a volume called "A Reader's Guide to Interpersonal Neurobiology" to be published by Norton. 